\title{
Expanding the phenotypic and genetic spectrum of TUBB8 in female infertility: Suggestions for genetic counseling
}

\author{
Wei Zheng ${ }^{1}$, Shuoping Zhang ${ }^{1}$, Pingyuan $\mathrm{Xie}^{2}$, Huiling $\mathrm{Hu}^{3}$, Jing Dai ${ }^{1}$, Xilin Xu ${ }^{1}$, Yifan \\ $\mathrm{Gu}^{1}$, Jia Wang ${ }^{1}$, Xiaofeng $\mathrm{Li}^{1}$, Yi Tang ${ }^{1}$, Hui Guo ${ }^{1}$, Liang $\mathrm{Hu}^{4}$, Fei Gong ${ }^{1}$, Guangxiu Lu${ }^{1}$, \\ and Ge $\operatorname{Lin}^{5}$ \\ ${ }^{1}$ Reproductive and Genetic Hospital CITIC Xiangya \\ ${ }^{2}$ Hunan Normal University School of Medicine \\ ${ }^{3}$ Central South University \\ ${ }^{4}$ Central South University Xiangya School of Medicine \\ ${ }^{5}$ CITIC Reproductive and Genetic Hospital Xiangya
}

April 28, 2020

\begin{abstract}
Tubulin beta 8 class VII (TUBB8), a primate oocyte/embryo-specific $\beta$-tubulin isotype, is a preferentially analyzed gene in the genetic diagnosis of infertile women. Studies have currently identified 47 variants of this gene. However, the evaluation of its pathogenicity and the resulting phenotypes vary, and this gene requires further study in order to be included in genetic counseling analyses. We have performed a whole exome sequencing (WES) screening of 385 infertile female subjects and 400 controls, identifying 29 TUBB8 variants (of which 23 were novel). These variants may be responsible for female infertility, especially in the case of embryonic arrest, and their identification further expands the variant spectrum of TUBB8. Our findings are the first to report a new phenotype of large polar body in TUBB8 variant patients and determine some recurrent variants that were specific for complete cleavage failure. We have also illustrated some phenotypes of TUBB8 variant patients and recommended using computational modeling approach to assess the pathogenicity of novel TUBB 8 variants, particularly for maternally inherited variants. Our studies will contribute in laying the foundation for the genetic counseling of infertile women in the future.
\end{abstract}

\section{INTRODUCTION}

Female infertility is a worldwide health problem that affects an estimated 48 million women (Mascarenhas, Flaxman, Boerma, Vanderpoel, \& Stevens, 2012). With the help of in vitro fertilization (IVF) and intracytoplasmic sperm injection (ICSI) techniques, many otherwise infertile couples have had babies successfully. However, there are still women who cannot establish a pregnancy, presenting recurrent IVF/ICSI failure due to oocyte meiotic arrest, fertilization failure, or embryonic arrest (Hart, 2016; Matzuk \& Lamb, 2008; Yatsenko \& Rajkovic, 2019).

It is known that human oocyte maturation is the first and key process of accurate cleavage competence, including nuclear maturation and cytoplasmic maturation (Moor, Dai, Lee, \& Fulka, 1998). Nuclear maturation can be easily verified by the extrusion of the first polar body (PB1), while cytoplasmic maturation involves various invisible events such as spindle assembly, organelle distribution, and maternal mRNA metabolism (Sha, Zhang, \& Fan, 2019).

Tubulin beta 8 class VII (TUBB8 ) is a highly conserved primate-specific $\beta$-tubulin isotype, which specifically participates in spindle assembly in oocytes and early embryos (Feng, Sang, et al., 2016). Feng et al . identified 
TUBB8 as the first disease-causing gene in oocyte meiotic arrest in 2006, and their two consecutive studies revealed a $37.2 \%$ prevalence of TUBB8 variants in a total of 43 patients (Feng, Sang, et al., 2016; Feng, Yan, et al., 2016). So far, 47 different variants (38 heterozygous variants and 8 homozygous variants) were reported as related to abnormalities in oocyte maturation, fertilization, and embryonic development (Chen et al., 2017; Chen et al., 2019; Feng, Sang, et al., 2016; Feng, Yan, et al., 2016; Huang et al., 2017; Lanuza-Lopez et al., 2020; Wang et al., 2018; Yuan et al., 2018). TUBB8 seems to be a preferentially analyzed gene in the genetic diagnosis of infertile women. However, evidence of the pathogenicity of TUBB8 variant has been evaluated through different strategies, such as in vitrofunctional verification, morphological observation of the spindle, pedigrees in segregation analysis, and the genetic detection of probands. The causal relationship between $T U B B 8$ variants and the various observed phenotypes thus requires further investigation, as does the spectrum of potential pathogenic TUBB8 variants.

In the present study, we identified 29 variants in TUBB8, of which 23 were novel, through whole exome sequencing (WES). These comprised 22 heterozygous variants and seven homozygous/compound heterozygous variants and may be responsible for female infertility in 32 of 385 independent families, and five of 29 variants in six families were maternally inherited of a total of 26 pedigree-analyzed families. We demonstrated various abnormalities in the oocytes, zygotes and embryos of TUBB8 variant patients. In addition, we suggested the possible causal relationship between large polar bodies and TUBB8variants in two families under the morphological observation of the spindle and reported the possible five variant hotspots for female infertility. Our findings are the first to suggest the possible pathogenesis of the large polar body, the variant hotspots extremely for complete cleavage failure and extend the variational and phenotypic spectrum of TUBB8 greatly. Our findings, together with the available knowledge on TUBB8 variants and female infertility, will potentially impact the genetic counseling of infertile women in future.

\section{METHODS}

\section{Clinical Samples}

385 Infertile female and 400 fertile controls in this study were recruited from the Reproductive and Genetic Hospital of CITIC-XIANGYA, China. All blood samples were donated for research after informed consent was obtained. This study was approved by the Ethics Committee of the Reproductive and Genetic Hospital of CITIC-XIANGYA (reference LL-SC-2017-009).

\section{WES and variant analysis}

Samples from the probands and fertile controls were subjected to WES. Variants were filtered as previously described (Zheng et al., 2020), by using the following criteria: (1) variants with minor allele frequencies (less than $1 \%$ in the Genome AD database); (2) exonic nonsynonymous or splice site variants, or coding insertions or deletions (indels); (3) mRNA and/or proteins that were highly or specifically expressed in oocytes; (4) coexistence in both probands but absence in controls.

\section{Sanger sequencing}

Specific primers flanking the variants in the TUBB8 gene were used for polymerase chain reaction (PCR) amplification with an ABI 3100 DNA analyzer (Applied Biosystems, Foster City, USA).

\section{Molecular modeling and evolutionary conservation analysis}

Wild-type (WT) and mutated TUBB8 were assessed using the SWISS-MODEL software (https://swissmodel.expasy.org) based on the 6e88.B.pdb template. Mutated TUBB8 was mapped to the atomic model using PyMol (http://www. pymol.org). Evolutionary conservation analysis was performed with MultiAlin (http://multalin.toulouse.inra.fr software.

\section{Polar body biopsy and chromosome analysis}

The PB1 was removed from the perivitelline space of the oocyte with a biopsy needle after laser dissection opening of the zona pellucida (ZP) and then placed into a $0.2-\mathrm{mL}$ microtube. Whole genome amplification 
was then performed using the PicoPLEX whole genome amplification kit (Rubicon Genomics, Ann Arbor, USA) to produce the DNA required for next-generation sequencing by an Illumina NextSeq 550 platform (Illumina, San Diego, USA) (Xie et al., 2019).

\section{RESULTS}

\section{Identification of TUBB8 pathogenic variants}

WES and Sanger sequencing were used for the identification of pathogenic variants. After filtering the data according to the criteria described in the Materials and methods section, we obtained TUBB8 (MIM: 616768; GenBank: NM_177987) variants from 32 of a total of 385 independent families. We identified 23 novel variants; 29 variants in total (Figure 1), including 25 missense variants, three frameshift insertion variants, and a non-frameshift insertion variant. All these were extremely rare (frequencies $<10^{-4}$ ) or absent in the databases, and were also not found in 400 healthy Chinese women with normal fertility (Table 1). Most of the variants $(22 / 29)$ were heterozygous and some of their inheritance patterns were unknown due to the absence of DNA samples from their parents. In addition, six families presented a recessive inheritance pattern, including families 3,4 , and 5 that were identified with a homozygous variant and families 1,6 , and 11 that were identified with compound heterozygous variant (Figure 1 and Table 1). We found some recurrent variants sites such as p.G308S, p.E108K, p.V353I, p.R262Q, p.R320H, and p.R391C; each of these variants was identified in two patients. p.E108K, p.R262Q, and p.R391C were previously reported as having a causal relationship with complete cleavage failure (Yuan et al., 2018), oocyte meiotic arrest (Feng, Sang, et al., 2016), and embryonic arrest (Chen et al., 2019), respectively. These aspects were consistent with the phenotypes observed of the same variant in our study. On the other hand, p.G308S and p.V353I were first presented as other two recurrent variant sites related to complete cleavage failure. Among the identified variants, we found five (p.A302T, p.A196T, p.T232S, p.R320H and p.A411D) in six families $(15,22,24,26$, 27 and 32 ) that were maternally inherited or also detected in fertile sister of the proband (Figure 1).

\section{Clinical characteristics and phenotypes of patients}

All 32 individuals from independent and hitherto uncharacterized families with primary female infertility underwent 2 to 3 failed IVF/ICSI attempts in our center or other hospitals. Detailed clinical information in our center including controlled ovarian hyperstimulation $(\mathrm{COH})$ protocol, oocyte maturity and morphology, as well as fertilization and embryo development outcomes is shown in Table 2. We observed that a significant proportion of oocytes showed a large polar body in affected individuals from Families 1 and $2(26.7 \%$ and $41.7 \%$, respectively), which had not yet been reported in TUBB8 variants (Figure 2A \& Table 2). In both patients, PB1 oocytes presented either an abnormal or undetectable spindle, and finally led to fertilization failure (Table 2); both the PB and the oocyte exhibited abnormal chromosome compositions, including partial chromosome trisomy or monosomy (Figure $2 \mathrm{~B}$ ). Since the phenotypes varied, we categorized them as oocyte meiotic arrest, abnormal fertilization, complete cleavage failure, and embryonic development arrest. The retrieved oocytes had been through germinal vesicle (GV)/metaphase I (MI) mixed arrest in Families 10-14; abnormal fertilization, mainly through high proportions of multi-pronuclei (MPN, Family 13) or one pronucleus (1PN, Family 15); and nearly all the embryos were arrested before the first cleavage, even after two extra days of cultivation (Families 3-9). All other families presented embryonic arrest, and although 1-2 usable embryos were produced in families 23, 26 and 29, there was a failure in their transfer. Among these individuals, we also observed various abnormalities in oocyte morphology, such as small or even unobvious PB1, vacuoles in ooplasm, dark plasm, empty ZP, and oocyte degeneration. In fertilized zygotes or embryos, there were problems of fragmentation or degeneration after ICSI embryo or asymmetric cleavage furrow formation or arrest between the 1 to 8-cell stages, accompanied or not by severe fragmentation.

\section{Impact of $T U B B 8$ variants on protein structure}

Among the novel missense variants (except that novel missense amino acid change occurring at the same position as another missense change has been reported), the amino acids at variant positions were highly conserved across different primate species except for p.Q15K and p.L42V. According to a three-dimensional (3D) structure of TUBB8 used to assess the effect of missense variants, the p.Q15K variant led to loss of the 
guanosine diphosphate (GDP) ligand, while other variants had no obvious effects on the protein structure. However, most of them were predicted to cause changes in the hydrogen bonds between mutated residues and nearby residues: some hydrogen bonds were absent after missense variants (p.Q15K, p.D88H, and p.R320H) and some new hydrogen bonds were produced (p.G141R, p.P182S, p.A196T, and p.R308S). The remaining variants did not present any changes in hydrogen bonds.

\section{DISCUSSION}

TUBB8 encodes a primate-specific beta-tubulin subunit in the oocyte and early embryo. An increasing number of studies indicate that TUBB8 heterozygous variants have dominant negative effects and that homozygous/compound-heterozygous variants disrupt spindle assembly and cause abnormalities in oocyte maturation, fertilization, and embryonic development (Chen et al., 2017; Chen et al., 2019; Feng, Sang, et al., 2016; Feng, Yan, et al., 2016; Huang et al., 2017; Lanuza-Lopez et al., 2020; Wang et al., 2018; Yuan et al., 2018). In this study, we reported 29 TUBB8 variants (23 novel) that further expand the variant spectrum of $T U B B 8$, extremely for the complete cleavage failure and embryonic arrest and indicate for the first time a possible causal relationship between large PB occurrence and TUBB8variants, thus also expanding phenotypic spectrum of TUBB8 variants.

So far, 14 of the total 47 TUBB 8 variants have been reported and their pathogenicity verified by TUBB 8 mutants in culture cells and/or mouse/human oocytes, showing abnormalities in microtubule phenotypes and nice variants have also showed abnormal spindle assembly under light and polarization microscopy (Figure $3 \mathrm{~A}$ ), which can certainly be used as genetic markers for genetic counseling in female infertility cases (Chen et al., 2017; Feng, Sang, et al., 2016; Feng, Yan, et al., 2016; Richards et al., 2015). Some variants conform to pedigrees in segregation analysis and some were only judged by the proband due to the unavailability of DNA samples from their parents (Chen et al., 2019), therefore the pathogenicity of these TUBB8 variants was not completely evaluated (Figure 3A). Two consecutive studies by Feng et al.revealed a significant prevalence $(37.2 \%)$ of TUBB8 variants in 43 patients (Feng, Sang, et al., 2016; Feng, Yan, et al., 2016), and in our study this frequency was about $8.3 \%(32 / 385)$; the difference between frequencies may be due to a bias in patient recruitment. Nevertheless, the judgement of pathogenic TUBB8 variants needs more consideration. Since TUBB8 is a primate-specific subunit with a high prevalence variant, building animal models for each of these variants is not trivial, and neither is their in vitro functional verification. The most effective methods include summarizing the reported variants and the phenotype of patients, showing someTUBB 8 variant hotspots such as p.R2, p.I4, p.G98, and p.S176. These variants should receive more attention even in the absence of powerful functional verification.

In our study, we also screened variant hotspots such as p.T143Dfs*12 (previously reported in two patients (Chen et al., 2019; Feng, Yan, et al., 2016)), p.G98R (previously reported in three patients (Chen et al., 2019; Wang et al., 2018)), p.R262Q (previously reported in one patient), and p.R391C (previously reported in one patient (Chen et al., 2019)); the observed phenotype was similar (Table 1 and Figure 3A). We also determined variant hotspots that were specific for complete cleavage failure, such as p.G308S (from Families 3 and 4), p.E108K (from Families 5 and 6, and previously reported in one patient with the same phenotype (Yuan et al., 2018)), p.V353I (from Families 7 and 8, and previously reported in two patients with the similar phenotype (Chen et al., 2017; Chen et al., 2019)). The retrieved oocytes were morphologically normal and successfully fertilized, but in our unpublished data we found that TUBB8 variant could explain 8 out of 10 patients that presented complete cleavage failure. Therefore, a TUBB 8 variant could aid in pre-diagnosis in order to avoid recurrent cleavage failures.

Human oocyte maturation includes nuclear maturation and cytoplasmic maturation. The nuclear maturation is verified by PB1 extrusion. Normally at MI, the spindle is first centrally positioned in the oocyte but then migrates to the cortex. This is a prerequisite for the asymmetric cleavage that produces the small polar body and the remaining larger competent secondary oocyte. However, in our patients (Families 1 and 2), the spindle presented various abnormalities such as spindle absence, altered shapes or localization errors. At the end of metaphase I, we observed that $26.7 \%(8 / 30)$ and $41.7 \%(5 / 12)$ of oocytes extruded abnormally large polar bodies, some even achieving mitotic-like cleavage and producing cells of equal size (Figure $2 \mathrm{~A}$ 
and Table 2). Choi et al. also observed the similar phenotype in maturing oocytes of Mos knockout mice oocytes, accompanied by the abnormal spindle, and they considered increased genetic instability may exist inMos knockout mice oocytes (Choi et al., 1996). Indeed, the abnormal spindle may cause the chromosome segregation error easily, and we demonstrated the chromosome separation error in the large PBs (Figure $2 \mathrm{~B})$.

Apart from some paternally inherited or de novo TUBB8variants, we also found five TUBB8 variants in six families that were maternally inherited. This type of maternally inherited has been previously explained by the possibility of an incomplete penetrance pattern or a modifier gene (Chen et al., 2019). Regarding the maternally inherited variants observed in this study, p.R320H coexisted in Families 26 and 27, and there were no obvious changes in the predicted protein structure or hydrogen bonds. p.R320H may therefore be a harmless variant, although this should be further investigated. Similarly, the remaining four maternally inherited variants were all predicted to have no changes, except for p.A196T. Among the three unknown inheritance pattern variants (p.E108K, p.L42V and p.G141R), p.L42V was the only one for which no changes were predicted. On the other hand, in segregation-analyzed families with these pedigrees, all variants were predicted to affect protein structure and/or hydrogen bonds. These results suggested that computational modeling is a meaningful method to evaluate the pathogenicity of novelTUBB8 variants in addition to frequency screening, pedigree and evolutionary conservation analysis. Of course, its need to further evaluated in combination with in vitro functional verification.

In our study, we exhibited representative pictures including abnormalities in oocyte morphology, fertilization, and embryonic development outcomes. These may give some reference significance in the genetic counseling of female infertility patients. The 34 patients from 32 families were classified as: 4 meiotic arrests, 2 large PB, 2 abnormal fertilizations, 8 complete cleavage failures, and 19 embryonic arrests. The average oocyte maturation rate in embryonic arrest patients is lower than that of others, and the percentage of severe fragmentation of the embryo is higher than that for others in our center (data not shown). We therefore recommend that $T U B B 8$ should be preferentially analyzed as a part of the genetic diagnosis of infertile female, together with the aforementioned phenotypes.

In conclusion, the 23 novel (of 29) variants encountered in TUBB8may contribute to female infertility especially through embryonic arrest and further expand the variant spectrum of TUBB8. Our findings are the first to suggest the possible pathogenesis of a large polar body and determine variant hotspots specific for complete cleavage failure. We have also exhibited representative pictures of TUBB8 variant patients, correlating our results and contributing to the foundation of the genetic counseling of infertile women in future.

\section{Acknowledgment}

We would like to thank the patients and their families for participating in this work and Editage (www.editage.cn) for English language editing.

\section{Data Availability Statement}

The datasets used and/or analysed during the current study available from the corresponding author on reasonable request.

\section{Conflicts of Interest}

The authors declare that they have no conflicts of interest to disclose.

\section{References}

Chen, B., Li, B., Li, D., Yan, Z., Mao, X., Xu, Y., . . . Wang, L. (2017). Novel mutations and structural deletions in TUBB8: expanding mutational and phenotypic spectrum of patients with arrest in oocyte maturation, fertilization or early embryonic development. Hum Reprod, 32 (2), 457-464. doi:10.1093/humrep/dew322

Chen, B., Wang, W., Peng, X., Jiang, H., Zhang, S., Li, D., . . . Wang, L. (2019). The comprehensive 
mutational and phenotypic spectrum of TUBB8 in female infertility.Eur J Hum Genet, 27 (2), 300-307. doi:10.1038/s41431-018-0283-3

Choi, T., Fukasawa, K., Zhou, R., Tessarollo, L., Borror, K., Resau, J., \& Vande Woude, G. F. (1996). The Mos/mitogen-activated protein kinase (MAPK) pathway regulates the size and degradation of the first polar body in maturing mouse oocytes.Proc Natl Acad Sci U S A, 93 (14), 7032-7035. doi:10.1073/pnas.93.14.7032

Feng, R., Sang, Q., Kuang, Y., Sun, X., Yan, Z., Zhang, S., . . . Wang, L. (2016). Mutations in TUBB8 and Human Oocyte Meiotic Arrest. N Engl J Med, 374 (3), 223-232. doi:10.1056/NEJMoa1510791

Feng, R., Yan, Z., Li, B., Yu, M., Sang, Q., Tian, G., . . . Wang, L. (2016). Mutations in TUBB8 cause a multiplicity of phenotypes in human oocytes and early embryos. J Med Genet, 53 (10), 662-671. doi:10.1136/jmedgenet-2016-103891

Hart, R. J. (2016). Physiological Aspects of Female Fertility: Role of the Environment, Modern Lifestyle, and Genetics. Physiol Rev, 96 (3), 873-909. doi:10.1152/physrev.00023.2015

Huang, L., Tong, X., Luo, L., Zheng, S., Jin, R., Fu, Y., . . . Liu, Y. (2017). Mutation analysis of the TUBB8 gene in nine infertile women with oocyte maturation arrest.Reprod Biomed Online, 35 (3), 305-310. doi:10.1016/j.rbmo.2017.05.017

Lanuza-Lopez, M. C., Martinez-Garza, S. G., Solorzano-Vazquez, J. F., Paz-Cervantes, D., GonzalezOrtega, C., Maldonado-Rosas, I., . . . Pina-Aguilar, R. E. (2020). Oocyte maturation arrest produced by TUBB8 mutations: impact of genetic disorders in infertility treatment. Gynecol Endocrinol , 1-6. doi:10.1080/09513590.2020.1725968

Mascarenhas, M. N., Flaxman, S. R., Boerma, T., Vanderpoel, S., \& Stevens, G. A. (2012). National, regional, and global trends in infertility prevalence since 1990: a systematic analysis of 277 health surveys. PLoS Med, 9 (12), e1001356. doi:10.1371/journal.pmed.1001356

Matzuk, M. M., \& Lamb, D. J. (2008). The biology of infertility: research advances and clinical challenges.Nat Med, 14 (11), 1197-1213. doi:10.1038/nm.f.1895

Moor, R. M., Dai, Y., Lee, C., \& Fulka, J., Jr. (1998). Oocyte maturation and embryonic failure.Hum Reprod Update, 4 (3), 223-236. doi:10.1093/humupd/4.3.223

Richards, S., Aziz, N., Bale, S., Bick, D., Das, S., Gastier-Foster, J., . . . Committee, A. L. Q. A. (2015). Standards and guidelines for the interpretation of sequence variants: a joint consensus recommendation of the American College of Medical Genetics and Genomics and the Association for Molecular Pathology. Genet Med, 17 (5), 405-424. doi:10.1038/gim.2015.30

Sha, Q. Q., Zhang, J., \& Fan, H. Y. (2019). A story of birth and death: mRNA translation and clearance at the onset of maternal-to-zygotic transition in mammalsdagger. Biol Reprod, 101 (3), 579-590. doi:10.1093/biolre/ioz012

Wang, A. C., Zhang, Y. S., Wang, B. S., Zhao, X. Y., Wu, F. X., Zhai, X. H., . . . Mei, S. Y. (2018). Mutation analysis of the TUBB8 gene in primary infertile women with arrest in oocyte maturation. Gynecol Endocrinol, 34 (10), 900-904. doi:10.1080/09513590.2018.1464138

Xie, P., Hu, L., Tan, Y., Gong, F., Zhang, S., Xiong, B., . . . Lin, G. (2019). Retrospective analysis of meiotic segregation pattern and interchromosomal effects in blastocysts from inversion preimplantation genetic testing cycles. Fertil Steril, 112 (2), 336-342 e333. doi:10.1016/j.fertnstert.2019.03.041

Yatsenko, S. A., \& Rajkovic, A. (2019). Genetics of human female infertilitydagger. Biol Reprod, 101 (3), 549-566. doi:10.1093/biolre/ioz084

Yuan, P., Zheng, L., Liang, H., Li, Y., Zhao, H., Li, R., . . . Wang, W. (2018). A novel mutation in the TUBB8 gene is associated with complete cleavage failure in fertilized eggs. J Assist Reprod Genet, 35 (7), 1349-1356. doi:10.1007/s10815-018-1188-3 
Zheng, W., Chen, L., Dai, J., Dai, C., Guo, J., Lu, C., . . . Lin, G. (2020). New biallelic mutations in PADI6 cause recurrent preimplantation embryonic arrest characterized by direct cleavage. $J$ Assist Reprod Genet, 37 (1), 205-212. doi:10.1007/s10815-019-01606-7

\section{Figure legends}

\section{Figure 1. Identification of variants in $T U B B 8$}

Pedigree analysis of 32 families with TUBB8 variants. Black squares with arrows indicate affected individuals. Sanger sequencing confirmation is shown in the right. Unavailable parental information is indicated by question marks.

Figure 2. Morphology of oocytes, zygotes, and embryos of control individuals and patients.

1. PB1 oocyte from control and patients in Families 1 and 2 were examined by light and polarization microscopy. The control oocyte has a visible spindle located near PB1, while it is only very weakly visible in the R $380 \mathrm{H}$ variant. No detectable spindle can be observed in the p.I91Hfs*34 and p.T143Dfs*12 compound-heterozygous variants.

2. Control PB1 had diploid chromosome copy numbers. The large PB and oocyte had abnormal chromosome compositions, including partial chromosome trisomy and partial chromosome monosomy.

3. The morphology of a control and affected individuals' oocytes on day 0 , zygotes on day 1 and embryos on day 3 after fertilization presenting various morphological abnormalities. Arrow indicated the PB1.

Figure 3. Bioinformatic analysis of $T U B B 8$ variants.

1. Summary of previously reported and of our TUBB8 variants. Black font represents all 47 variants, red font represents the 29 variants of our study. All variants include the inherited pattern and hierarchy of evidence. The significance of digital indication is explained in the chart.

2. Conservation analysis of mutated amino acids in 5 different primate species. All the variant sites are highly conserved except for Q15K and L42V.

3. Protein conformation predictions of variants in TUBB8 . Red dashed lines represent hydrogen bonds, indicated by the black arrowhead. 

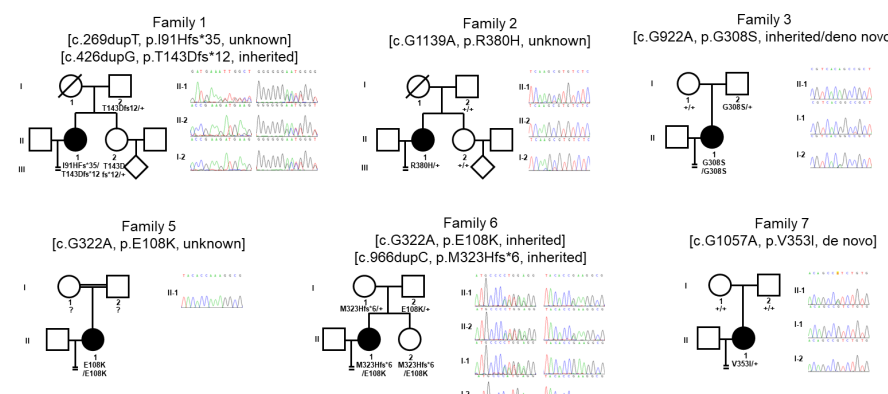

Family 4
[c.G922A, p. G308S, inherited]

[c.269dupt, p.191Hfs*35, unknown]
[c.426dup G, p.T143Dfs" 12 , inherited]
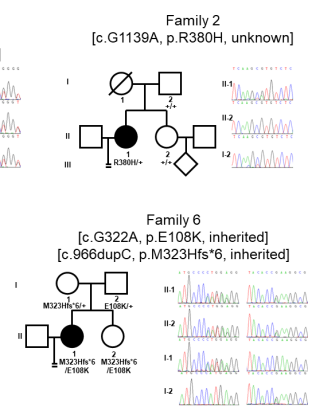

Family 7
[c.G1057A, p.V3531, de novo]

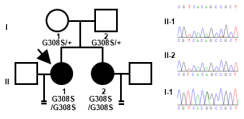

Family 9
[c.C1072T, p.P358S, unknown]
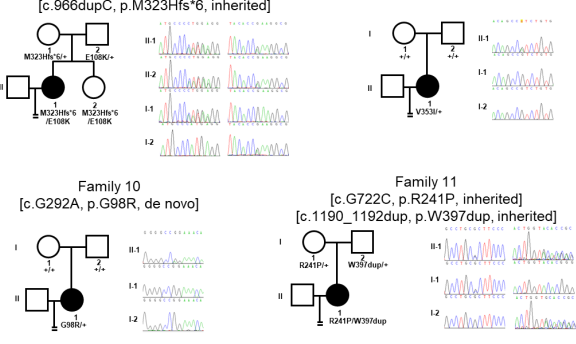

Family 8
[c.G1057A, p.V3531, inherited]

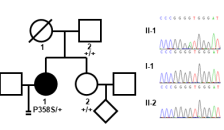

[c.G292A, p.G98R, de novo]

Family 11

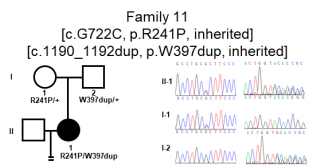

"

Family 12
[c.G785A, p.R262Q, de novo]

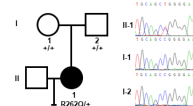

Family 13
[c.G785A, p.R262Q, de novo]

$\bigcirc_{1+1}^{2} \prod_{2}^{2}$

" 口-10

Family 14
[c.G1172T, p.R391L, de novo]

(1)

Family 15
[c.G904A, p.A302T, unknown]
1

(1)

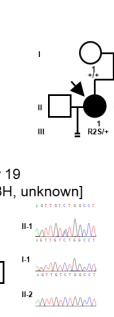

Family 16
[c.G6T, p.R2S, inherited]

thowlyny
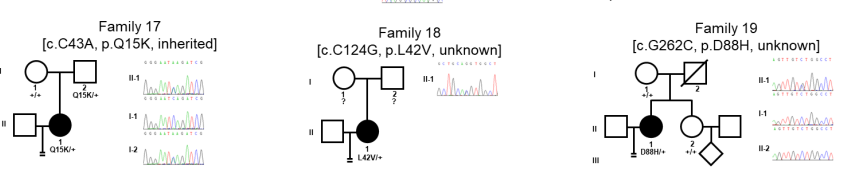

Family 21
[c.C544T, p.P182S, inherited]
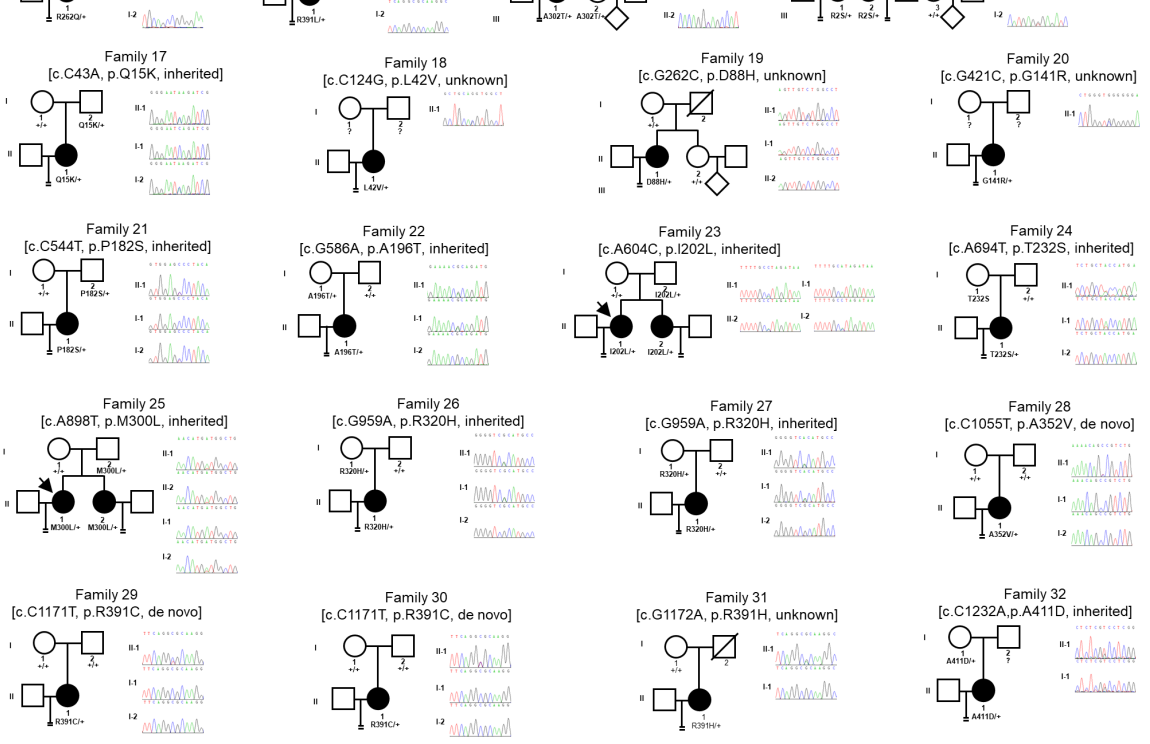

Family 28
[c.C1055T, p.A352V, de novo]

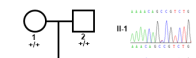

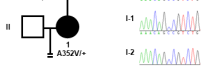

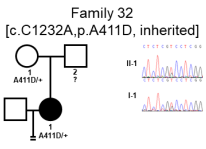


A
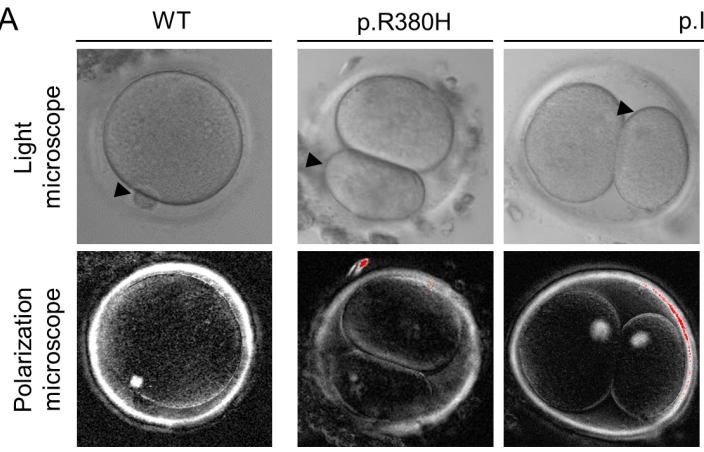

p.191fs*34\& p.T143Dfs*12

B

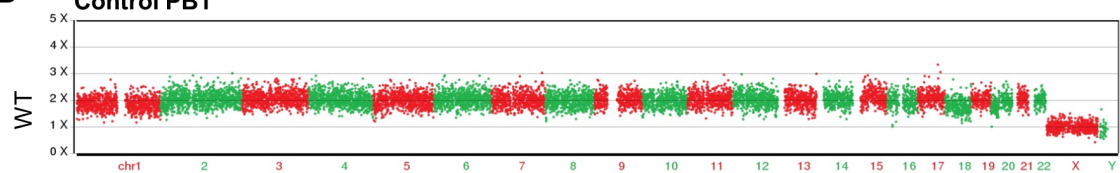
Large PB1

${ }_{4 \times}^{5 \times}$

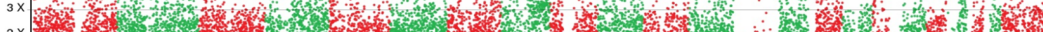

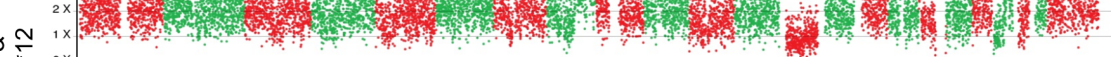

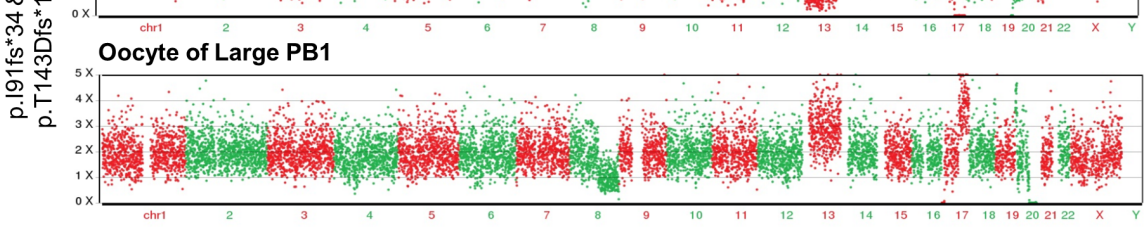

C
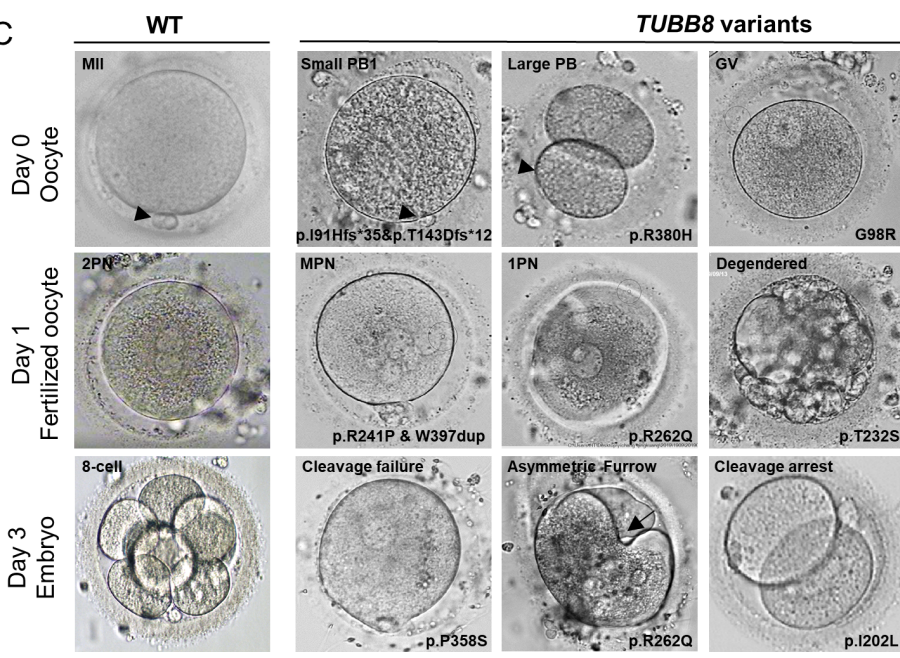
Cleavage failüre $\zeta_{5}$
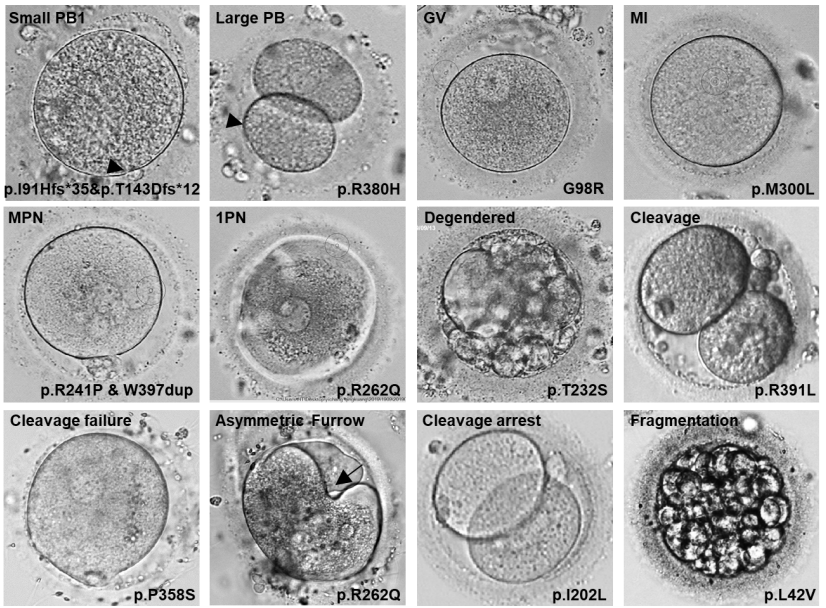
A

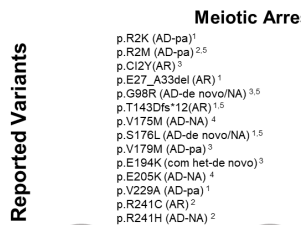
p.V255M (AD-de novolNA)
p. R262W(AD-de novo) $)^{\prime}$ (a) $262 \mathrm{(AD}$-de novo)

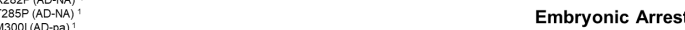
Abnormal Fertilization

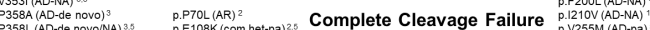

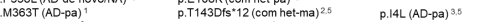

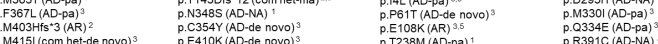
(

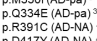

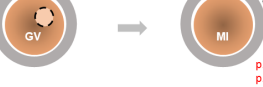

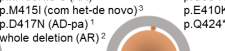

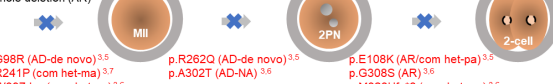

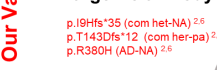

B

$\begin{array}{lllllll} & \text { p.Q15K } & \text { p.L42V } & \text { p.D88H } & \text { p.G141R } & \text { p.P182S } & \text { p.A196T p.I202L } \\ \text { Homo sapiens } & \text { QCGNQIGAK } & \text { GDSHLQLER } & \text { VFRPDNFIF } & \text { SLGGGTGSG } & \text { TVVEPYNAT } & \text { NADETFCID } \\ \text { Pan troglodytes } & \text { QCGNQIGAK } & \text { GDSHLQLER } & \text { VFRPDNFIF } & \text { SLGGGTGSG } & \text { TVVEPYNAT } & \text { NADETFCID } \\ \text { Gorilla gorilla gorilla } & \text { QCGNQIRAK } & \text { GDSHLQLER } & \text { VFRPDSFIF } & \text { CLGGGTGSG } & \text { TVVEPYNAT } & \text { NADETFCID } \\ \text { Macaca fascicularis } & \text { QCGNRMGV } & \text { GDNQGQLEH } & \text { VFRPDNIF } & \text { SLGGGGSG } & \text { TVVEPYNAT } & \text { NADETFCID } \\ \text { Papio anubis } & \text { QCGNRIGVK } & \text { GYNQGQLER } & \text { VFRPDNFIF } & \text { SLGGGTGSG } & \text { TVVEPYNAT } & \text { NADETFCID } \\ \text { Consensus } & \text { QCGNRIGVK } & \text { GDNQGQLER } & \text { VFRPDNFIF } & \text { SLGGGTGSG } & \text { TVVEPYNAT } & \text { NADETFCID } \\ & \text { p.T232S p.A302T p.G308S } & \text { p.R320H } & \text { p.R380H } & \text { p.A411D } \\ \text { Homo sapiens } & \text { LVSATMSGV } & \text { AACDPRHGR } & \text { IFRGRMPMR } & \text { ELFKRVSEQ } & \text { EFTEAESNM } \\ \text { Pan troglodytes } & \text { LVCATMSGV } & \text { AACDPRHGR } & \text { IFRGRMPMR } & \text { ELFKRVSEQ } & \text { EFTEAESNM } \\ \text { Gorilla gorilla gorilla } & \text { LVSATMSGV } & \text { AACDPRHGR } & \text { IFRGRMPMR } & \text { ELFKRVSEQ } & \text { EFTEAESNM } \\ \text { Macaca fascicularis } & \text { LVSATMSGV } & \text { AACDPRHGR } & \text { IFRGRITTR } & \text { ELFKRVSE } & \text { EFTQAESNM } \\ \text { Papio anubis } & \text { LVSATMSGV } & \text { AACDPRHGR } & \text { IFRGRITMR } & \text { ELFKRSEQ } & \text { EFTQAESNM } \\ \text { Consensus } & \text { LVSATMSGV } & \text { AAC. PRHGR } & \text { IFRGRITMR } & \text { ELFKRVSEQ } & \text { EFT.AESNM }\end{array}$

C

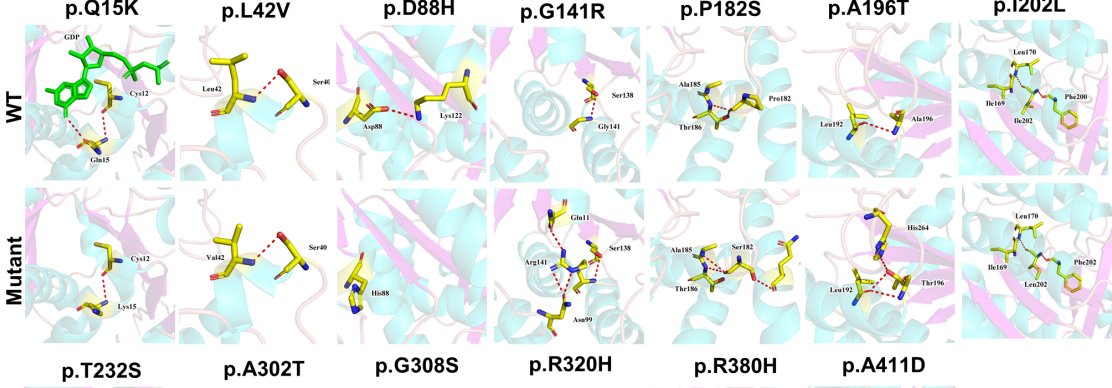

(n)

(1) 